\title{
Dynamical properties of a dissipative discontinuous map: A scaling investigation
}

\author{
R. Aguilar-Sánchez, ${ }^{1}$ Edson D. Leonel, ${ }^{2}$ and J. A. Méndez-Bermúdez ${ }^{3}$ \\ ${ }^{1}$ Facultad de Ciencias Químicas, Benemérita Universidad Autónoma de Puebla, Puebla 72570, \\ Mexico \\ ${ }^{2}$ Departamento de Física, UNESP - Univ Estadual Paulista, Av. 24A, 1515, Bela Vista, \\ 13506-900 Rio Claro, SP, Brazil \\ ${ }^{3}$ Instituto de Física, Benemérita Universidad Autónoma de Puebla, Apartado Postal J-48, \\ Puebla 72570, Mexico
}

\begin{abstract}
The effects of dissipation on the scaling properties of nonlinear discontinuous maps are investigated by analyzing the behavior of the average squared action $\left\langle I^{2}\right\rangle$ as a function of the $n$-th iteration of the map as well as the parameters $K$ and $\gamma$, controlling nonlinearity and dissipation, respectively. We concentrate our efforts to study the case where the nonlinearity is large; i.e., $K \gg 1$. In this regime and for large initial action $I_{0} \gg K$, we prove that dissipation produces an exponential decay for the average action $\langle I\rangle$. Also, for $I_{0} \cong 0$, we describe the behavior of $\left\langle I^{2}\right\rangle$ using a scaling function and analytically obtain critical exponents which are used to overlap different curves of $\left\langle I^{2}\right\rangle$ onto an universal plot. We complete our study with the analysis of the scaling properties of the deviation around the average action $\omega$.
\end{abstract}

Keywords: Scaling, Discontinuous map, Dissipation

PACS: 05.45.-a, 05.45.Pq

\section{Introduction}

Nonlinear maps are discrete-time dynamical systems which emerge as local approximations to continuous-time motion, directly from a dynamical problem, or from the successive intersections of a continuous-time trajectory with a phase space section. Nonlinear maps have been extensively used to model dynamical systems in diverse knowledge areas: from maths and physics to finance and social 
sciences, passing through biology and chemistry. However, in physics, maps are of special relevance since they can be directly derived from, and transformed to, Hamiltonian systems; in this case they are named as canonical maps. Remarkably, there are analytical results available that describe nonlinear canonical maps; namely, KAM theorem and Poincaré-Birkhoff theorem. See for example [1].

One of the most studied canonical maps is Chirikov's standard map (CSM), introduced in Ref. [2] as a Poincaré surface of section of the kicked rotor (a prototype model for quantum chaos). CSM is an area preserving two-dimensional (2D) map for action and angle variables $(I, \theta)$

$$
T_{\mathrm{CSM}}:\left\{\begin{array}{l}
I_{n+1}=I_{n}+K \sin \left(\theta_{n}\right), \\
\theta_{n+1}=\theta_{n}+I_{n+1}, \quad \bmod (2 \pi)
\end{array} .\right.
$$

CSM, which is identified as a continuous map due to the sine function, describes the situation when nonlinear resonances are equidistant in phase space which corresponds to a local description of dynamical chaos [1]. Due to this property various dynamical systems and maps can be locally reduced to map (1). Thus, CSM describes the universal and generic behavior of nearly-integrable Hamiltonian systems with two degrees of freedom having a divided phase space composed of stochastic motion bounded by invariant tori (known as KAM scenario) [1].

CSM develops two dynamical regimes separated by the critical parameter $K_{\mathrm{C}} \approx 0.971635 \ldots\left[1,2,2,3,4,5,6,6\right.$, 7]. When $K<K_{\mathrm{C}}$, known as regime of weak nonlinearity, the motion is mainly regular with regions of stocasticity where the action $I$ is bounded by KAM surfaces. At $K=K_{\mathrm{C}}$, the last KAM curve is destroyed and the transition to global stocasticity takes place. Then, for $K>K_{\mathrm{C}}$, regime of strong nonlinearity, $I$ becomes unbounded and increases diffusively.

In particular, in Ref. [8] a scaling analysis of CSM was performed by studying the average value of the squared action $\left\langle I^{2}\right\rangle$ as a function of $K$ and the $n$-th iteration of the map. There, the following scaling law was reported:

$$
\left\langle I^{2}\right\rangle \propto n^{\alpha} K^{\beta}
$$

where $\alpha \approx 2$ for $K \ll K_{\mathrm{C}}$ and small $n$ while $\alpha \approx 1$ for $K \gg K_{\mathrm{C}}$ and large $n$, with $\beta \approx 2$ in both cases.2 The scaling of Eq. (2) turned out to be more general and has

\footnotetext{
${ }^{1}$ Note that in the regime of weak nonlinearity, $K<K_{\mathrm{C}}$, the action $I$ is bounded; however, the scaling of Eq. (2) is observed before $\left\langle I^{2}\right\rangle$ saturates.

${ }^{2}$ It is also relevant to mention that the scaling of Eq. (2) is valid regardless the value of $I_{0}$ : $I_{0}<K$ (even $I_{0}=0$ ) or $I_{0}>K$. However, for $I_{0}>K$ the behavior of $\left\langle I^{2}\right\rangle$ versus $n$ becomes richer; we refer the reader to Ref. [8] for details.
} 
also been observed for other dynamical systems that can be represented locally (but not exclusively) by the standard map such as the Fermi-Ulam model [9, 10, 11, 12], time-dependent potential wells [13], waveguide billiards [14]; among other nonlinear systems [15, 16].

In this paper we consider as dynamical model a dissipative version of the discontinuous CSM. We will seek to understand and describe the behavior of the squared action as a function of the control parameters of the map as well as the time. To this end we will make use of analytical treatments supported by extensive numerical simulations. For the regime of large nonlinearity in the presence of dissipation, when the dynamics starts with small initial action, the curves we obtain for the averaged square action show a clear growth that is stopped when the curves reach a regime of saturation. The saturation is produced by the presence of the dissipation. Indeed it is a consequence of the fact that the determinant of the Jacobian matrix is smaller than one. Such property leads to the existence of attractors in the phase space. Since such attractors are far away from infinity, the trajectories can not diffuse without limit, in contrast to the nondissipative case. So this is a clear transition from unlimited to limited diffusion in the action. For the best knowledge of the authors, we found analytically the exponents describing such a dynamical regime for the first time. Our results are totally supported by large scale numerical simulations. In addition, when the initial action is large enough, say few orders of magnitude larger than the nonlinearity, we prove analytically that the decay of the action is exponential. Also, numerical simulations are used to validate the analytical findings, giving total support for our results. The procedures we used in this paper are general and may be extended to different types of nonlinear models, particularly to higher dimensional mappings.

\section{Model and numerical procedure}

Indeed, even though CSM describes the universal behavior of area-preserving continuous maps, other class of Hamiltonian dynamical systems are represented by the so called discontinuous map [17]

$$
T_{\text {DCSM }}:\left\{\begin{array}{l}
I_{n+1}=I_{n}+K \sin \left(\theta_{n}\right) f\left(\theta_{n}\right), \\
\theta_{n+1}=\theta_{n}+\tau I_{n+1}, \quad \bmod (2 \pi)
\end{array},\right.
$$

where

$$
f\left(\theta_{n}\right)=\operatorname{sgn}\left[\cos \left(\theta_{n}\right)\right] .
$$


There are several physical systems that can be described by discontinuous maps, including 2D billiard models like the stadium billiard [18, 19] and polygonal billiards [20, 21]. The origin of the discontinuity in map (3) are the sudden translations of the action under the system dynamics.

In the same way as CSM, mapping (3) is known to have two different dynamical regimes delimited by the critical parameter $K_{\mathrm{C}}=1 / \tau$ [17]; nevertheless, both of them are diffusive. The regimes $K<K_{\mathrm{C}}$ and $K>K_{\mathrm{C}}$ are known as slow diffusion and quasilinear diffusion regimes, respectively. On the one hand, the main difference between CSM and map (3) is that for $K<K_{\mathrm{C}}$ the later does not show regular behavior. In fact, due to the discontinuity of $f(\theta)$, KAM theorem is not satisfied and map (3) does not develop the KAM scenario. Since for any $K \neq 0$ the dynamics of map (3) is diffusive, a single trajectory can explore the entire phase space. However, in the slow diffusion regime the dynamics is far from being stochastic due to the sticking of trajectories along cantori (fragments of KAM invariant tori). On the other hand, for $K>K_{\mathrm{C}}$ map (3) shows diffusion similar to that of CSM.

Following the same general procedure reported in Ref. [8], in Ref. [22] it was found that the scaling of $\left\langle I^{2}\right\rangle$ for discontinuous maps when $K \ll K_{\mathrm{C}}$ and $K \gg K_{\mathrm{C}}$ obeys the same scaling laws, in the appropriate limits, than CSM in the regimes of weak and strong nonlinearity, respectively. However, due to absence of KAM tori, it was observed that $\left\langle I^{2}\right\rangle$ is described as a power law of the type $\left\langle I^{2}\right\rangle \propto n K^{\beta}$; which applies in both regimes (for large enough $n$ ) with $\beta \approx 5 / 2$ when $K \ll K_{\mathrm{C}}$ and $\beta \approx 2$ for $K \gg K_{\mathrm{C}}$.

Since the purpose of this work is to study the effects of dissipation on the scaling properties of a discontinuous map, to consider a dissipative dynamics we propose the following mapping

$$
T_{\text {DDCSM }}:\left\{\begin{array}{l}
I_{n+1}=(1-\gamma) I_{n}+K \sin \left(\theta_{n}\right) f\left(\theta_{n}\right), \\
\theta_{n+1}=\theta_{n}+\tau I_{n+1}, \quad \bmod (2 \pi)
\end{array},\right.
$$

where $\gamma \in[0,1]$ is the dissipation parameter and the function $f\left(\theta_{n}\right)$ is given by Eq. (4). If $\gamma=0$ in (5) the Hamiltonian area-preserving discontinuous map of Eq. (3) is recovered. We shall consider $0<\gamma<1$. Given the determinant of the Jacobian matrix is $1-\gamma$, the system is area preserving only when $\gamma=0$. For mapping (5) we study some of the properties for the scaling of the average value of the squared action variable $\left\langle I^{2}\right\rangle$ and of the deviation around the average action $\omega$, as a function of $n, K, I_{0}$, and $\gamma$.

We compute $\left\langle I^{2}\right\rangle$ for map (5) following two steps: First we calculate the aver- 
age squared action over the orbit associated with the initial condition $j$ as

$$
\left\langle I_{n, j}^{2}\right\rangle=\frac{1}{n+1} \sum_{i=0}^{n} I_{i, j}^{2},
$$

where $i$ refers to the $i$-th iteration of the map. Then, the average value of $I^{2}$ is defined as the average over $M$ independent realizations of the map (by randomly choosing values of $\theta_{0}$ ):

$$
\left\langle I^{2}\right\rangle\left(n, K, I_{0}, \gamma\right)=\frac{1}{M} \sum_{j=1}^{M}\left\langle I_{n, j}^{2}\right\rangle .
$$

Then, we define the average standard deviation of $I$ as

$$
\omega\left(n, K, I_{0}, \gamma\right)=\frac{1}{M} \sum_{j=1}^{M} \sqrt{\left\langle I_{n, j}^{2}\right\rangle-\left\langle I_{n, j}\right\rangle^{2}},
$$

where, in analogy with Eq. (6), $\left\langle I_{n, j}\right\rangle=(n+1)^{-1} \sum_{i=0}^{n} I_{i, j}$.

In the following, without lose of generality, we set $\tau=1$. In our simulations, for each combination of parameters $\left(K, I_{0}, \gamma\right)$, we consider an ensemble of 1000 different initial random phases uniformly distributed in the interval $0<\theta_{0}<2 \pi$. A larger ensemble leads to qualitatively the same results.

\section{Results. Case $K \gg 1$}

We divide our investigation in two parts, both considering $K \gg 1$. The first is devoted to $I_{0} \cong 0$ while the second takes into account $I_{0} \gg 1$.

3.1. $I_{0} \cong 0 \ll K$

Because of the symmetry of the phase space from both positive $(I>0)$ and negative $(I<0)$ sides, the average $\langle I\rangle$ is not the most convenient variable to look at when $I_{0} \cong 0$. Instead, we consider $\left\langle I^{2}\right\rangle$. Figure 1(a) shows plots of $\left\langle I^{2}\right\rangle$ as a function of $n$ for several values of $\gamma$ and two different values of $K$ : $K=10^{2}$ and $K=10^{3}$. For fixed $K$, we observe that the curves $\left\langle I^{2}\right\rangle$ vs. $n$ show two different regimes: a growth regime, for small iteration values, and a saturation regime marked by a constant plateau $I_{\text {sat }}^{2}$, for large enough $n$. The transition from growth to saturation is characterized by the crossover iteration number $n_{x}^{(1)}$. We notice that different values of $K$ make the curves of $\left\langle I^{2}\right\rangle$ to grow parallel to each other 

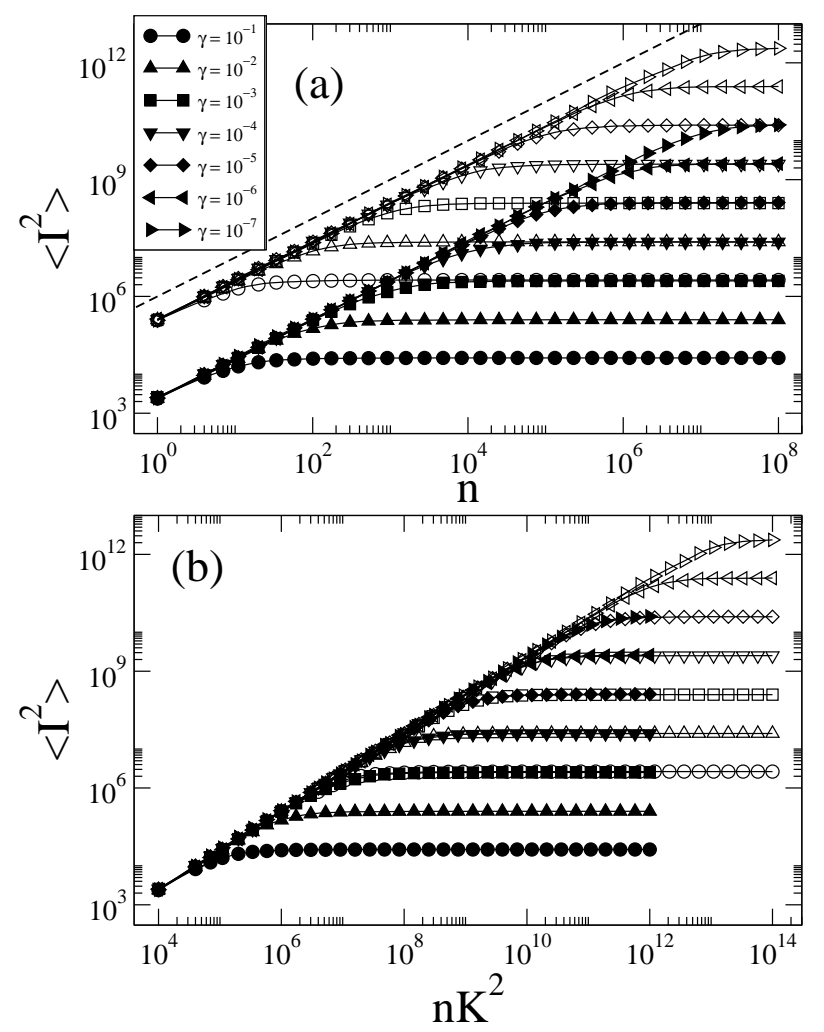

Figure 1: $\left\langle I^{2}\right\rangle$ as a function of (a) $n$ and (b) $n K^{2}$ for the map of Eq. (5) when $K \gg 1$ and $I_{0} \ll K$. Full symbols (open symbols) correspond to $K=10^{2}\left(K=10^{3}\right) . I_{0}=K / 100$ was used. The dashed line in (a) proportional to $n$ is plotted to guide the eye. In (b), after the transformation $n \rightarrow n K^{2}$, all curves grow together.

with the same slope, leading us to believe that $n$ is not a good scaling variable. In fact, by applying the transformation $n \rightarrow n K^{2}$ the growth part of all curves $\left\langle I^{2}\right\rangle$ vs. $n$ coalesce, as shown in Fig. 1(b).

Based on the behavior shown in Fig. 1 we propose the following scaling hypotheses for $\left\langle I^{2}\right\rangle$ :

(i) $\left\langle I^{2}\right\rangle\left(n K^{2}, K, \gamma\right) \propto\left(n K^{2}\right)^{\beta}$, for $n \ll n_{x}^{(1)}$, where $\beta$ is an accelerating exponent;

(ii) $I_{\text {sat }}^{2} \propto K^{\alpha_{1}} \gamma^{\alpha_{2}}$, for $n \gg n_{x}^{(1)}$, where both $\alpha_{1}$ and $\alpha_{2}$ are scaling exponents;

(iii) $n_{x}^{(1)} K^{2} \propto K^{z_{1}} \gamma^{z_{2}}$, with $z_{1}$ and $z_{2}$ representing dynamical exponents.

As a consequence of these scaling hypotheses, we can describe the behavior 
of $\left\langle I^{2}\right\rangle$ using a homogeneous function of the type

$$
\left\langle I^{2}\right\rangle\left(n K^{2}, K, \gamma\right)=\ell\left\langle I^{2}\right\rangle\left(\ell^{a} n K^{2}, \ell^{b} K, \ell^{c} \gamma\right),
$$

where $\ell$ is a scaling factor and $a, b$, and $c$ are scaling exponents. In fact, since $\ell$ is an arbitrary scaling factor, we can make the following independent choices:

- $\ell^{a} n K^{2}=1$.

Then, substituting $\ell=\left(n K^{2}\right)^{-1 / a}$ into (9) leads to

$$
\left\langle I^{2}\right\rangle\left(n K^{2}, K, \gamma\right)=\left(n K^{2}\right)^{-1 / a}\left\langle I^{2}\right\rangle_{1}\left(\left(n K^{2}\right)^{-b / a} K,\left(n K^{2}\right)^{-c / a} \gamma\right),
$$

where $\left\langle I^{2}\right\rangle_{1}\left(\left(n K^{2}\right)^{-b / a} K,\left(n K^{2}\right)^{-c / a} \gamma\right)=\left\langle I^{2}\right\rangle\left(1,\left(n K^{2}\right)^{-b / a} K,\left(n K^{2}\right)^{-c / a} \gamma\right)$ is assumed to be constant for $n \ll n_{x}^{(1)}$. By comparing Eq. (10) with the first scaling hypotheses, we end up with $\beta=-1 / a$.

The accelerating exponent $\beta$ can be obtained by performing power-law fittings to the curves $\left\langle I^{2}\right\rangle$ vs. $n$ for $n \ll n_{x}^{(1)}$. Indeed, we found $\beta=1$ from several different simulations, see the dashed line in Fig. 1 (a).

- $\ell^{b} K=1$.

After substitution of $\ell=K^{-1 / b}$ into Eq. (9) we get

$$
\left\langle I^{2}\right\rangle\left(n K^{2}, K, \gamma\right)=K^{-1 / b}\left\langle I^{2}\right\rangle_{2}\left(K^{-a / b} n K^{2}, K^{-c / b} \gamma\right),
$$

where $\left\langle I^{2}\right\rangle_{2}\left(K^{-a / b} n K^{2}, K^{-c / b} \gamma\right)=\left\langle I^{2}\right\rangle\left(K^{-a / b} n K^{2}, 1, K^{-c / b} \gamma\right)$ is assumed to be constant for $n \gg n_{x}^{(1)}$. By comparing Eq. (11) with the second scaling hypotheses we obtain that $\alpha_{1}=-1 / b$.

In Fig. 2(a) we plot $I_{\text {sat }}^{2}$ vs. $K$ for fixed $\gamma$. There, the power law $I_{\text {sat }}^{2} \propto K^{\alpha_{1}}$ is clearly observed. A power-law fitting to the data provides $\alpha_{1}=2$.

- $\ell^{c} \gamma=1$.

By substituting $\ell=\gamma^{-1 / c}$ into Eq. (9) we obtain

$$
\left\langle I^{2}\right\rangle\left(n K^{2}, K, \gamma\right)=\gamma^{-1 / c}\left\langle I^{2}\right\rangle_{3}\left(\gamma^{-a / c} n K^{2}, \gamma^{-b / c} K\right)
$$

where $\left\langle I^{2}\right\rangle_{3}\left(\gamma^{-a / c} n K^{2}, \gamma^{-b / c} K\right)=\left\langle I^{2}\right\rangle\left(\gamma^{-a / c} n K^{2}, \gamma^{-b / c} K, 1\right)$ is supposed to be constant for $n \gg n_{x}^{(1)}$. Thus, from the comparison of Eq. (12) with the second scaling hypotheses, we get $\alpha_{2}=-1 / c$. 

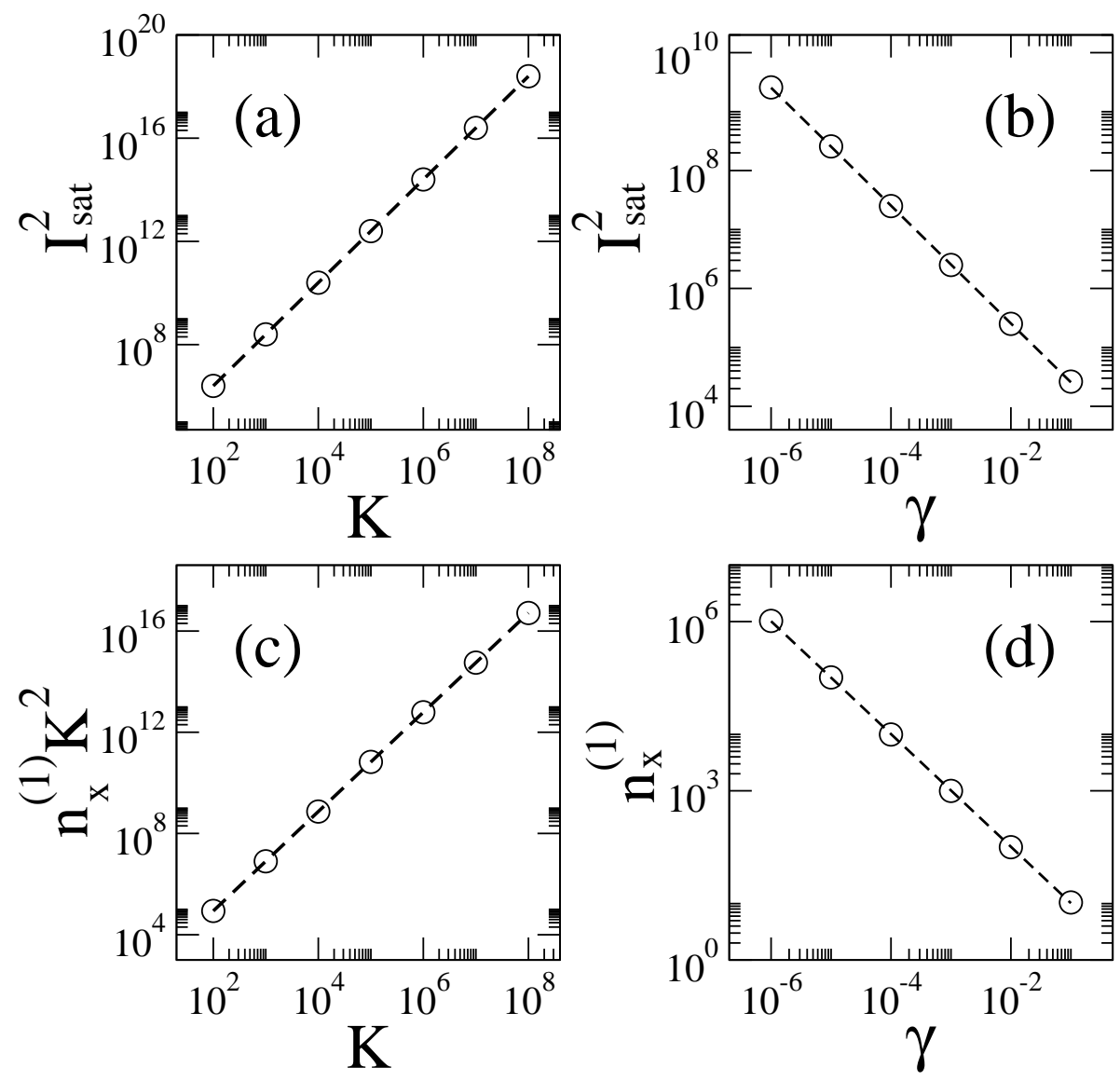

Figure 2: $I_{\text {sat }}^{2}$ as a function of (a) $K$ and (b) $\gamma$. (c) $n_{x}^{(1)} K^{2}$ as a function of $K$. (d) $n_{x}^{(1)}$ as a function of $\gamma$. In (a) and (c) $\gamma$ was set to $10^{-3}$. In (b) and (d) $K$ was set to $10^{2}$. In all cases $I_{0}=K / 100$ was used. Power law fittings to the data demonstrate the scalings (a) $I_{\text {sat }}^{2} \propto K^{\alpha_{1}}$ with $\alpha_{1}=2$, (b) $I_{\text {sat }}^{2} \propto \gamma^{\alpha_{2}}$ with $\alpha_{2}=-1$, (c) $n_{x}^{(1)} K^{2} \propto K^{z_{1}}$ with $z_{1}=2$, and (d) $n_{x}^{(1)} \gamma^{z_{2}}$ with $z_{2}=-1$; see dashed lines in the corresponding panels. 
Then, in Fig.2 (b) we present $I_{\text {sat }}^{2}$ vs. $\gamma$ for fixed $K$. From this figure the power law $I_{\text {sat }}^{2} \propto \gamma^{\alpha_{2}}$ is evident. Here, we obtain $\alpha_{2}=-1$ from the power-law fitting of the data.

To obtain the dynamical exponents $z_{1}$ and $z_{2}$, we can use the three different expressions for $\ell$ we obtained above: (1) $\ell=\left(n K^{2}\right)^{\beta}$, (2) $\ell=K^{\alpha_{1}}$, and (3) $\ell=\gamma^{\alpha_{2}}$. Indeed by comparing (1) with (2) we get $\left(n K^{2}\right)^{\beta} \propto K^{\alpha_{1}}$, for fixed $\gamma$. Therefore, $n K^{2} \propto K^{\alpha_{1} / \beta}$, leading to the scaling law

$$
z_{1}=\frac{\alpha_{1}}{\beta} .
$$

Comparing now (2) and (3) for a fixed $K$, we obtain that $\left(n K^{2}\right)^{\beta} \propto \gamma^{\alpha_{2}}$, producing the scaling law

$$
z_{2}=\frac{\alpha_{2}}{\beta} .
$$

Therefore, since we already know the values of $\alpha_{1,2}$ and $\beta$ we can compute the values of the dynamical exponents as: $z_{1}=2$ and $z_{2}=-1$. Numerical simulations confirm these values for $z_{1,2}$, as shown in Figs. 2(c) and2(d), where we plot $n_{x}^{(1)} K^{2}$ vs. $K$ and $n_{x}^{(1)}$ vs. $\gamma$, respectively.

The exponents defined above can be used on the transformations $\left\langle I^{2}\right\rangle \rightarrow$ $\left\langle I^{2}\right\rangle /\left(K^{\alpha_{1}} \gamma^{\alpha_{2}}\right)$ and $n \rightarrow n K^{2} /\left(K^{z_{1}} \gamma^{z_{2}}\right)$ to overlap the curves $\left\langle I^{2}\right\rangle$ vs. $n$ onto a single and therefore universal curve. This is shown in Fig. 3(a), where we clearly observe that all curves $\left\langle I^{2}\right\rangle /\left(K^{\alpha_{1}} \gamma^{\alpha_{2}}\right)$ vs. $n K^{2} /\left(K^{z_{1}} \gamma^{z_{2}}\right)$ fall one on top of the other for several combinations of $K$ and $\gamma$.

We found a similar behavior for the observable $\omega$, which is the deviation around the average action. Since $\omega^{2} \propto\left\langle I^{2}\right\rangle$, we could conclude that

(i) $\omega \propto\left(n K^{2}\right)^{\beta / 2}$, for $n \ll n_{x}^{(1)}$;

(ii) $\omega_{\text {sat }} \propto K^{\alpha_{1} / 2} \gamma^{\alpha_{2} / 2}$, for $n \gg n_{x}^{(1)}$;

(iii) $n_{x}^{(\omega)} \propto K^{z_{1}} \gamma^{z_{2}}$.

Here, the exponents $\beta, \alpha_{1,2}$, and $z_{1,2}$ are the same exponents we defined for the scaling of $\left\langle I^{2}\right\rangle$.

The validation of the scaling hypotheses for $\omega$ is shown in Fig. 3(b) where we show the collapse of all curves $\omega /\left(K^{\alpha_{1}} \gamma^{\alpha_{2}}\right)$ vs. $n K^{2} /\left(K^{z_{1}} \gamma^{z_{2}}\right)$ onto a single universal curve for several combinations of $K$ and $\gamma$. 

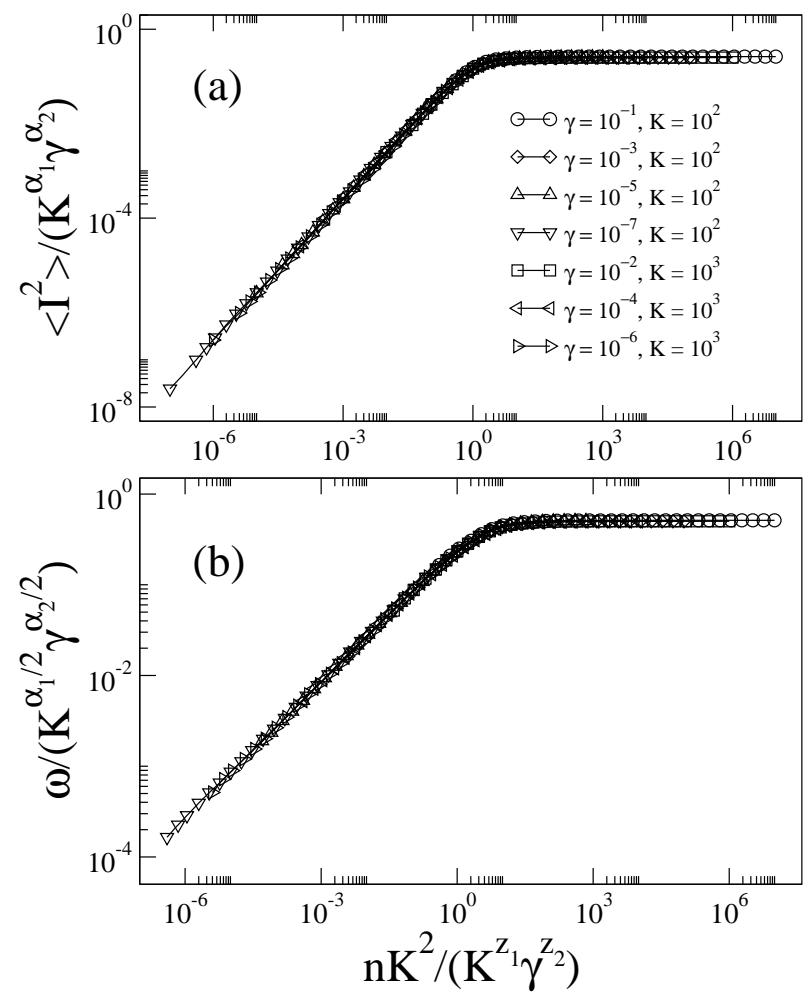

Figure 3: (a) $\left\langle I^{2}\right\rangle$ and (b) $\omega$ as a function of $n K^{2}$ for the map of Eq. (5) when $K \gg 1$ and $I_{0} \ll K$. The curves are properly scaled on both axis to fall on top of a universal plot. Several values of $K$ and $\gamma$ were used with $I_{0}=K / 100$. 


\subsubsection{Analytical finding of the critical exponents $\beta, \alpha_{1}$, and $\alpha_{2}$}

In this subsection we give analytical arguments to support the values we obtained numerically for the critical exponents $\beta, \alpha_{1}$, and $\alpha_{2}$.

On the one hand, we know that $\left\langle I_{n}\right\rangle=0$ due to the symmetry of the phase space. Hence, the dispersion of $I$, indeed $\omega$, must behave as $\omega \propto \sqrt{n}$, therefore $\left\langle I^{2}\right\rangle \propto n$ for $n \ll n_{x}^{(1)}$. Using the transformation $n \rightarrow n K^{2}$ as made previously we obtain $\left\langle I^{2}\right\rangle \propto n K^{2}$. A comparison with the scaling hypothesis (i) leads to $\beta=1$.

On the other hand, at equilibrium, i.e. at saturation, we must have $\left\langle I_{n+1}^{2}\right\rangle=$ $\left\langle I_{n}^{2}\right\rangle=I_{\text {sat }}^{2}$, hence

$$
\begin{aligned}
\left\langle I_{n+1}^{2}\right\rangle= & \left\langle\left[(1-\gamma) I_{n}+K \sin \left(\theta_{n}\right) f\left(\theta_{n}\right)\right]^{2}\right\rangle \\
= & (1-\gamma)^{2}\left\langle I_{n}^{2}\right\rangle+K^{2}\left\langle\sin ^{2}\left(\theta_{n}\right) f^{2}\left(\theta_{n}\right)\right\rangle \\
& +2(1-\gamma) K\left\langle I_{n}\right\rangle\left\langle\sin \left(\theta_{n}\right) f\left(\theta_{n}\right)\right\rangle \\
= & (1-\gamma)^{2}\left\langle I_{n}^{2}\right\rangle+K^{2}\left\langle\sin ^{2}\left(\theta_{n}\right) f^{2}\left(\theta_{n}\right)\right\rangle=\left\langle I_{n}^{2}\right\rangle=I_{\text {sat }}^{2} .
\end{aligned}
$$

The term $2(1-\gamma) K\left\langle I_{n}\right\rangle\left\langle\sin \left(\theta_{n}\right) f\left(\theta_{n}\right)\right\rangle$ above was eliminated because both $\left\langle I_{n}\right\rangle=$ 0 (given the symmetry of the phase space) and $\left\langle\sin \left(\theta_{n}\right) f\left(\theta_{n}\right)\right\rangle=0$. Therefore, $\left(1-2 \gamma+\gamma^{2}\right) I_{\text {sat }}^{2}+K^{2}\left\langle\sin ^{2}\left(\theta_{n}\right) f^{2}\left(\theta_{n}\right)\right\rangle=I_{\text {sat }}^{2}$ or $\gamma(\gamma-2) I_{\text {sat }}^{2}=-K^{2}\left\langle\sin ^{2}\left(\theta_{n}\right) f^{2}\left(\theta_{n}\right)\right\rangle$, which leads to

$$
I_{\text {sat }}^{2}=\frac{\left\langle\sin ^{2}\left(\theta_{n}\right) f^{2}\left(\theta_{n}\right)\right\rangle}{2-\gamma} K^{2} \gamma^{-1}=C K^{2} \gamma^{-1},
$$

where we have set $\left\langle\sin ^{2}\left(\theta_{n}\right) f^{2}\left(\theta_{n}\right)\right\rangle /(2-\gamma)=C$ as a constant. From Eq. (15) we conclude that $I_{\text {sat }}^{2} \propto K^{2} \gamma^{-1}$, meaning that $\alpha_{1}=2$ and $\alpha_{2}=-1$, see scaling (ii). We just recall that these values for $\alpha_{1}$ and $\alpha_{2}$ have been already numerically validated in Figs. 2(a) and 2(b), respectively.

\section{2. $I_{0} \gg K$ : Exponential decay of action}

Let us now consider the case of large initial action: $I_{0} \gg K$. For large $I$, the variable $\theta_{n+1}$ becomes strongly uncorrelated with $\theta_{n}$. It therefore makes the function $\sin \left(\theta_{n}\right) \operatorname{sgn}\left[\cos \left(\theta_{n}\right)\right]$ to fluctuate very fast. Then, for sufficiently small $K$, as compared to $I_{0}$, we can write $I_{n} \approx(1-\gamma)^{n} I_{0}$ that for $n \gg 1$ gets the form

$$
I_{n} \approx I_{0} \exp (-\gamma n)
$$

which predicts the exponential decay of the action with a decay rate equal to the dissipation parameter $\gamma$. 
To verify Eq. (16) in Fig. (4) (a) we plot $\langle I\rangle /\left|I_{0}\right|$ as a function of $n$ for several combinations of $I_{0}$ and $\gamma$. We are including Eq. (16) as red dashed lines. It is clear from this figure that Eq. (16) reproduces well the numerically obtained data, mainly for large $\gamma$ : that is, when $\gamma \rightarrow 0$ the damping is weak and do not produce a clean exponential decay of $I$; see for example the curves for $\gamma=10^{-6}$ in Fig. (4) (a) (right-most curves) which show a decay that is not well described by Eq. (16). Moreover, due to the excellent correspondence between theory and numerics for large enough $\gamma$, in Fig. (4) (b) we show the overlap of the curves $\langle I\rangle / I_{0}$ when plotted as a function of $x \equiv \gamma n$.

Even though Eq. (16) provides a suitable global description of $\langle I\rangle$ vs. $n$, we stress that the oscillations of $\langle I\rangle$ around zero for large $n$ do not disappear when $n \rightarrow \infty$; mainly for $\gamma \rightarrow 0$ where the oscillations are in fact quite large. This fact should manifest itself in the behavior of $\left\langle I^{2}\right\rangle$ and $\omega$ making them grow (instead of decrease) in an intermediate interval of $n$, as will be shown below. This is indeed expected since the approach developed above is valid for $I \gg K$ only, which may not be satisfied when $n$ is large and $\gamma$ small.

Now, let us explore the behavior of $\left\langle I^{2}\right\rangle$. In Fig. [5(a) we plot $\left\langle I^{2}\right\rangle$ as a function of $n$ for several values of $\gamma$. We have used two values of $I_{0}: I_{0}=10^{4}$ and $I_{0}=10^{5}$. According to this figure, the behavior of $\left\langle I^{2}\right\rangle$ as a function of $n$ is as follows. During the first iteration steps, since $K$ is small as compared to $I_{0},\left\langle I^{2}\right\rangle$ does not change significantly as a function of $n$; so $\left\langle I^{2}\right\rangle$ remains approximately equal to $I_{0}^{2}$ up to a crossover iteration number $n_{x}$. Then, when $n>n_{x},\left\langle I^{2}\right\rangle$ shows two different behaviors. On the one hand, for small $\gamma$, let us say for $\gamma<\gamma_{\mathrm{C}},\left\langle I^{2}\right\rangle$ increases as a function of $n$ up to a second crossover iteration number $n_{x x}$. On the other hand, for large $\gamma$, say for $\gamma \geq \gamma_{\mathrm{c}},\left\langle I^{2}\right\rangle$ decreases as a function of $n$ up to $n_{x x}$. Finally, in both cases, once $n>n_{x x},\left\langle I^{2}\right\rangle$ saturates and gets a constant value that we call $I_{\text {sat }}^{2}$. Moreover, we have found that $\gamma_{\mathrm{C}} \approx 10^{-4}$.

In the following, the crossover iteration numbers $n_{x}$ and $n_{x x}$ are labeled with (2) or (3) when they correspond to $\gamma \geq \gamma_{\mathrm{C}}$ or $\gamma<\gamma_{\mathrm{C}}$, respectively.

Since we have previously concluded that Eq. (16), $I_{n} \approx I_{0} \exp (-\gamma n)$, describes well the behavior of $\langle I\rangle$ vs. $n$ for large $\gamma$, we should try to use this fact to describe the behavior of $\left\langle I^{2}\right\rangle$ vs. $n$ when $\gamma \geq \gamma_{c}$; i.e., when $\left\langle I^{2}\right\rangle$ decreases in the interval $n_{x}^{(2)}<n<n_{x x}^{(2)}$. To this end we substitute Eq. (16) into Eq. (6) to write

$$
\left\langle I^{2}\right\rangle \approx \frac{1}{n} \int_{0}^{n} I_{x}^{2} d x=\frac{I_{0}^{2}}{2 \gamma n}[1-\exp (-2 \gamma n)] .
$$



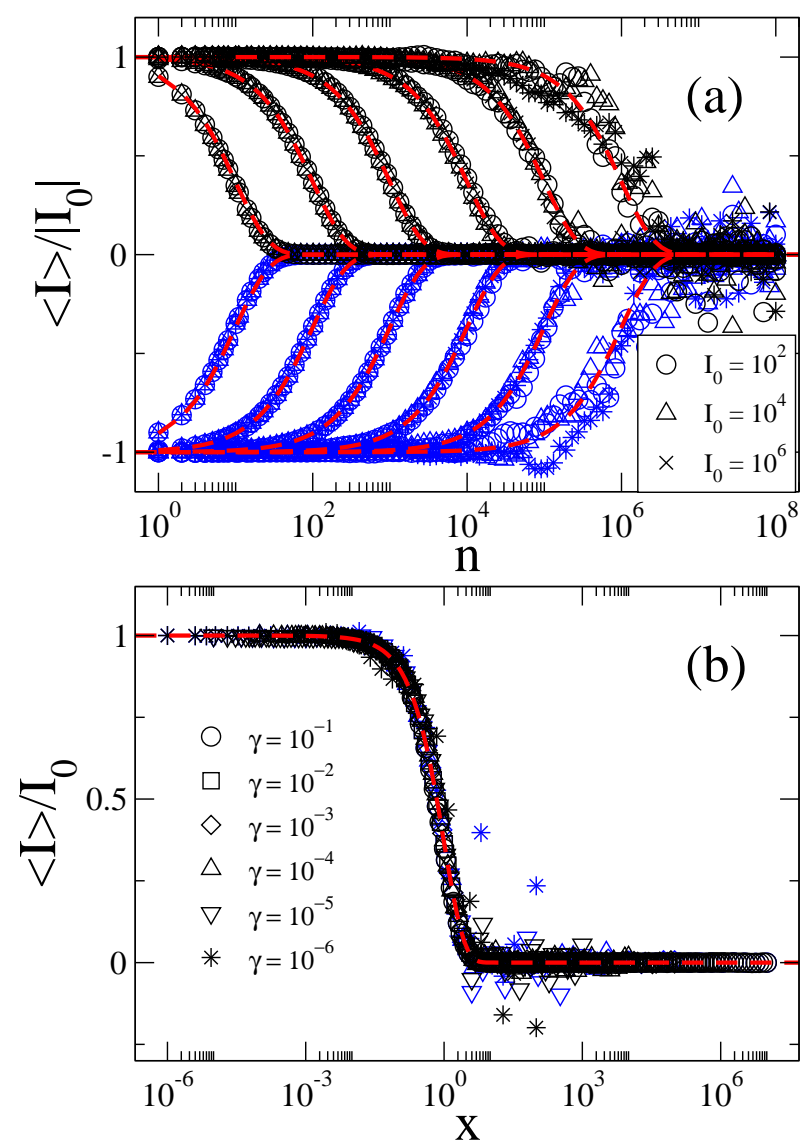

Figure 4: (a) $\langle I\rangle /\left|I_{0}\right|$ as a function of $n$ and (b) $\langle I\rangle / I_{0}$ as a function of $x$, where $x=\gamma n$, for the map of Eq. (5) when $K \gg 1$ and $I_{0} \gg K . K=I_{0} / 100$ was used. Black (blue) symbols correspond to $I_{0}>0\left(I_{0}<0\right)$. In (a) the groups of symbols correspond to $\gamma=10^{-1}, 10^{-2}, 10^{-3}, 10^{-4}, 10^{-5}$, and $10^{-6}$, from left to right. In (b) all symbols correspond to $I_{0}=10^{3}$. Red dashed lines in (a) $[(b)]$ are $\pm \exp (-\gamma n)[\exp (-x)]$. 

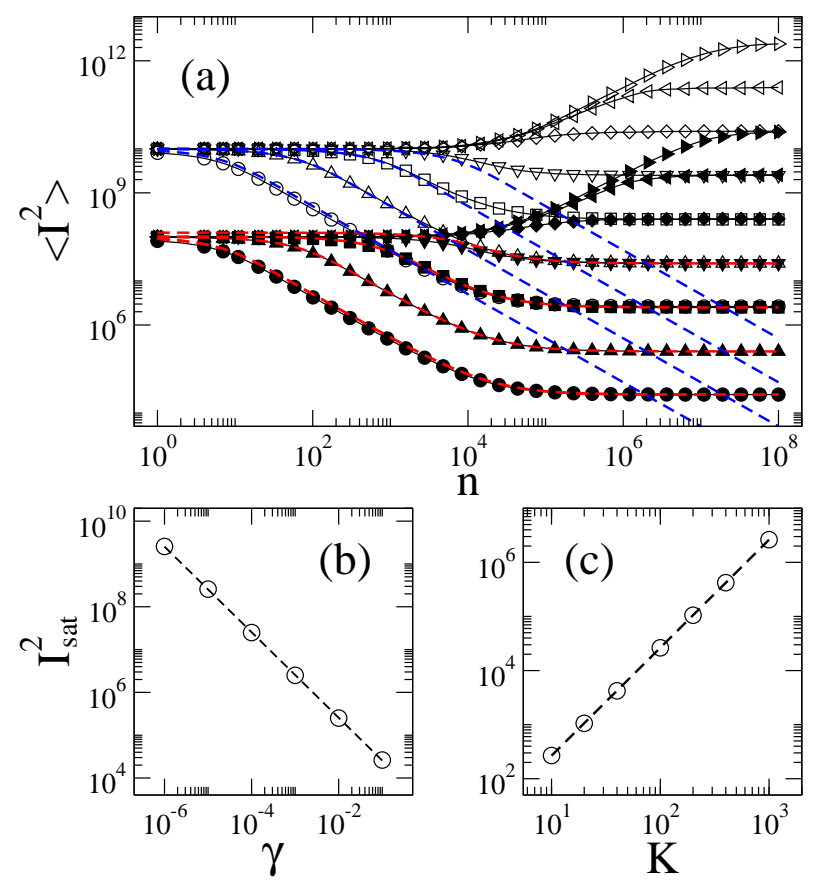

Figure 5: (a) $\left\langle I^{2}\right\rangle$ as a function of $n$ for the map of Eq. (5) when $K \gg 1$ and $I_{0} \gg K$. Full (open) symbols correspond to $K=10^{2}\left(K=10^{3}\right) . I_{0}=100 K$ was used. The curves in each set, full symbols and open symbols, correspond to $\gamma=10^{-1}, 10^{-2}, 10^{-3}, 10^{-4}, 10^{-5}, 10^{-6}$, and $10^{-7}$, from bottom to top. Blue [Red] dashed lines are Eq. (17) [Eq. (18)]. (b) [(c)] $I_{\text {sat }}^{2}$ as a function of $\gamma[K]$ for $K=10^{2}$ and $I_{0}=10^{4}\left[\gamma=10^{-1}\right.$ and $\left.I_{0}=10^{4}\right]$. The power-law fittings in (b-c), see dashed lines, demonstrate the scalings $I_{\text {sat }}^{2} \propto \gamma^{\alpha_{2}}$ and $I_{\text {sat }}^{2} \propto K^{\alpha_{1}}$ with $\alpha_{2}=-1$ and $\alpha_{1}=2$, respectively. 
In fact, Eq. (17) reproduces very well the numerical data for $\left\langle I^{2}\right\rangle$ vs. $n$ when $\gamma \geq \gamma_{\mathrm{C}}$ and $n<n_{x x}^{(2)}$, as demonstrated in Fig. 5(a) with the blue dashed lines (we only plotted Eq. (17) for the set of curves corresponding to $I_{0}=10^{5}$ to avoid the saturation of the figure). Moreover, since for $n>n_{x x}^{(2)},\left\langle I^{2}\right\rangle$ saturates, we propose the following correction to Eq. (17):

$$
\left\langle I^{2}\right\rangle \approx \frac{I_{0}^{2}}{2 \gamma n}[1-\exp (-2 \gamma n)]+I_{\text {sat }}^{2},
$$

Also, in Fig. 5(a) we plot Eq. (18) in red dashed lines now for the set of curves corresponding to $I_{0}=10^{4}$ observing an excellent correspondence with the numerical data.

We want to stress that from Eq. (18) we can extract the dependencies of $\left\langle I^{2}\right\rangle$ in the regimes bounded by $n_{x}^{(2)}$ and $n_{x x}^{(2)}$. When $n<n_{x}^{(2)}$, since both $\gamma$ and $n$ are small, $\left\langle I^{2}\right\rangle \approx\left(I_{0}^{2} / 2 \gamma n\right)[1-(1-2 \gamma n)]=I_{0}^{2}$; when $n_{x}^{(2)}<n<n_{x x}^{(2)},\left\langle I^{2}\right\rangle \approx I_{0}^{2} / 2 \gamma n$; while for $n>n_{x x}^{(2)},\left\langle I^{2}\right\rangle \approx I_{\text {sat }}^{2}$.

In addition, from Figs. 5 (b-c) we conclude that

$$
I_{\text {sat }}^{2} \propto K^{\alpha_{1}} \gamma^{\alpha_{2}}
$$

with $\alpha_{1}=2$ and $\alpha_{2}=-1$, which are in fact the same scaling exponents we found for $I_{\text {sat }}^{2}$ in the case $I_{0} \ll K$. It is interesting to mention that the scalings given in Eq. (19) do not depend whether $\gamma \geq \gamma_{\mathrm{C}}$ or $\gamma<\gamma_{\mathrm{C}}$.

We can also estimate $n_{x}^{(2)}\left[n_{x x}^{(2)}\right]$ by noticing that at $n_{x}^{(2)}\left[n_{x x}^{(2)}\right]$ the curves $\left\langle I^{2}\right\rangle \approx I_{0}^{2}$ $\left[\left\langle I^{2}\right\rangle \approx I_{0}^{2} / 2 \gamma n\right]$ and $\left\langle I^{2}\right\rangle \approx I_{0}^{2} / 2 \gamma n\left[\left\langle I^{2}\right\rangle \approx I_{\text {sat }}^{2}\right]$ should coincide. This gives

$$
n_{x}^{(2)}=(2 \gamma)^{-1} \text {, }
$$

and

$$
n_{x x}^{(2)}=\frac{I_{0}^{2}}{2 I_{\text {sat }}^{2} \gamma} \propto K^{-2} .
$$

Concerning the case $\gamma<\gamma_{\mathrm{C}}$, we observe, by the use of power-law fittings in the interval $n_{x}^{(3)}<n<n_{x x}^{(3)}$, that $\left\langle I^{2}\right\rangle \propto n$ (not shown here). This, together with $\left\langle I^{2}\right\rangle \propto K^{2}$, see the first line in Eq. (15), gives

$$
\left\langle I^{2}\right\rangle \propto n K^{2}
$$



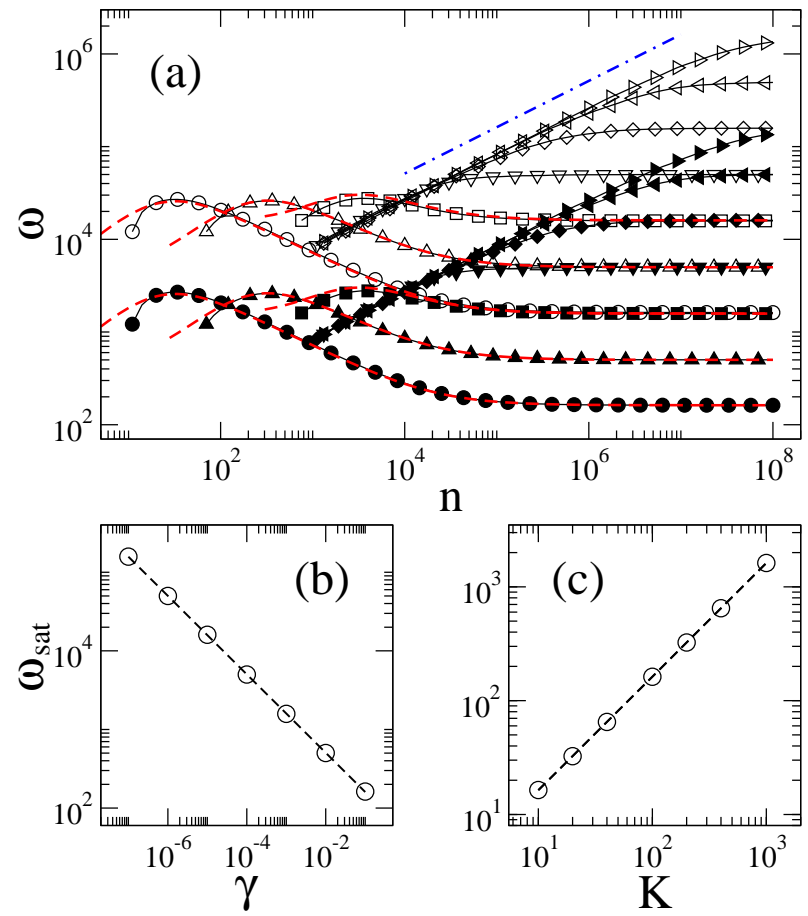

Figure 6: (a) $\omega$ as a function of $n$ for the map of Eq. (5) when $K \gg 1$ and $I_{0} \gg K$. Same parameters as in Fig. [5 a). The blue dot-dashed line proportional to $\sqrt{n}$ is plotted to guide the eye. Red dashed lines are Eq. (20). (b) [(c)] $\omega_{\text {sat }}$ as a function of $\gamma[K]$ for $K=10^{2}$ and $I_{0}=10^{4}\left[\gamma=10^{-1}\right.$ and $\left.I_{0}=10^{4}\right]$. The power-law fittings in (b-c), see dashed lines, demonstrate the scalings $I_{\text {sat }}^{2} \propto \gamma^{\alpha_{2} / 2}$ and $I_{\text {sat }}^{2} \propto K^{\alpha_{1} / 2}$ with $\alpha_{2}=-1$ and $\alpha_{1}=2$, respectively.

for $n_{x}^{(3)}<n<n_{x x}^{(3)}$. By matching this dependency with $\left\langle I^{2}\right\rangle \approx I_{0}^{2}$ for $n<n_{x}^{(3)}$ and $\left\langle I^{2}\right\rangle \approx I_{\text {sat }}^{2}$ when $n>n_{x x}^{(3)}$, we estimate that

$$
n_{x}^{(3)} \propto I_{0}^{2} K^{-2}
$$

and

$$
n_{x x}^{(3)} \propto \frac{I_{\text {sat }}^{2}}{K^{2}} \propto \gamma^{-1}
$$

Finally, let us recall that the increase of $\left\langle I^{2}\right\rangle$ in the interval $n_{x}^{(3)}<n<n_{x x}^{(3)}$ is related to the non-negligible oscillations of $I$, around its mean, when $\gamma$ is small (see Fig. 4).

To complete our analysis, in Fig. 6(a) we plot $\omega$ as a function of $n$ for the same parameter values used in Fig. 5(a). In correspondence with $\left\langle I^{2}\right\rangle$, we found 
two different behaviors for $\omega$ : (i) for large $\gamma, \gamma>\gamma_{\mathrm{C}}, \omega$ shows a fast increase as a function of $n$, reaches a maximum, and then decreases to approach a saturation value $\omega_{\text {sat }}$; (ii) for small $\gamma, \gamma<\gamma_{\mathrm{C}}, \omega$ increases proportional to $\sqrt{n}$ up to a crossover iteration number $n_{x}^{\omega}$, then for $n>n_{x}^{\omega}, \omega$ becomes the constant $\omega_{\text {sat }}$.

Since for $\gamma>\gamma_{\mathrm{C}}$ we have an expression to describe $\left\langle I^{2}\right\rangle$ vs. $n$ [see Eq. (18)] and $\omega^{2} \equiv\left\langle I^{2}\right\rangle-\langle I\rangle^{2}$ with $\left\langle I_{n}\right\rangle=(n+1)^{-1} \sum_{i=0}^{n} I_{i}$ [see Eq. (8)], we can write

$$
\omega^{2} \approx \frac{I_{0}^{2}}{2 \gamma n}[1-\exp (-2 \gamma n)]-\left(\frac{I_{0}}{\gamma n}\right)^{2}[1-\exp (-\gamma n)]^{2}+I_{\text {sat }}^{2},
$$

where we have used Eq. (16) to get

$$
\langle I\rangle \approx \frac{1}{n} \int_{0}^{n} I_{x} d x=\frac{I_{0}}{\gamma n}[1-\exp (-\gamma n)] .
$$

Then, in Fig. 6(a) we plot Eq. (20), as red dashed lines, for $\gamma>\gamma_{\mathrm{C}}$ and observe very good correspondence with the numerical data.

From Eq. (20) we can also see, for $n>n_{x}^{\omega}$, that

$$
\omega_{\mathrm{sat}} \approx \sqrt{I_{\mathrm{sat}}^{2}} \propto K^{\alpha_{1} / 2} \gamma^{\alpha_{2} / 2}
$$

compare with Eq. (19). This scaling of $\omega_{\text {sat }}$ is verified in Figs. 6(b-c). Also, we have concluded that

$$
n_{x}^{\omega} \approx n_{x x}^{(3)} \propto \gamma^{-1}
$$

(not shown). Then, we observe the collapse of the curves $\omega / \omega_{\text {sat }}$ when plotted as a function of (i) $n$, for $\gamma>\gamma_{\mathrm{C}}$; and (ii) $n \gamma$, for $\gamma<\gamma_{\mathrm{C}}$. See Fig.7.

\section{Conclusions}

In this paper we studied the effects of dissipation on the scaling properties of nonlinear discontinuous maps by analyzing the average value of the squared action $\left\langle I^{2}\right\rangle$ and the deviation around the average action $\omega$ as a function of $n$ ( $n$ being the $n$th iteration of the map). To this end we incorporate dissipation, parametrized by $\gamma$, into a two-dimensional discontinuous map whose dynamics is characterized by the stocasticity parameter $K$; see Eq. (5). We divide our investigation in two parts, both considering the case of $K \gg 1$ : The first is devoted to a small initial action, $I_{0} \cong 0$, while the second takes into account $I_{0} \gg 1$.

For $I_{0} \cong 0$, we described the behavior of $\left\langle I^{2}\right\rangle$ using a scaling function and analytically obtain critical exponents which are used to overlap different curves of 

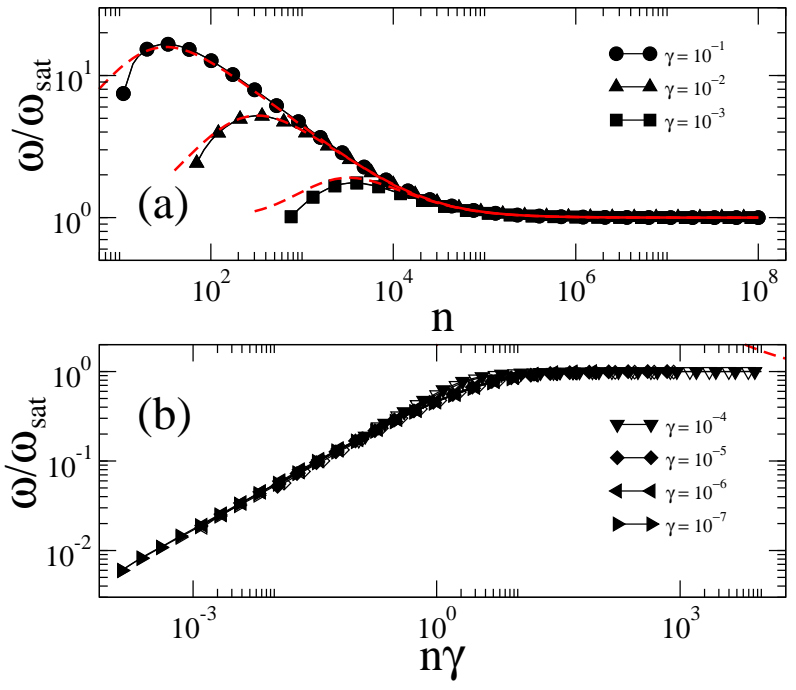

Figure 7: (a) $\omega / \omega_{\text {sat }}$ as a function of (a) $n$ when $\gamma>\gamma_{\mathrm{C}}$ and (b) $n \gamma$ when $\gamma<\gamma_{\mathrm{C}}$ for the map of Eq. (5) when $K \gg 1$ and $I_{0} \gg K$. Same data as in Fig. 6(a). Red dashed lines are Eq. (20). $\gamma_{\mathrm{C}} \approx 10^{-4}$

$\left\langle I^{2}\right\rangle$ and $w$ onto universal plots; see Fig. 3. For $I_{0} \gg K$, we found that dissipation produces the exponential decay of the average $I$ as a function of $n$. This fact, allowed us to write analytical expressions describing $\left\langle I^{2}\right\rangle$ and $w$; see Eqs. (18) and (20), respectively. Our results are summarized in Table 1 .

Finally, we want to add that due to the similar behavior of Chirikov's standard map (CSM) in the regime of strong nonlinearity $K>K_{\mathrm{C}} \sim 1$ and of the discontinuous map of Eq. (3) in the quasilinear diffusion regime $K>K_{\mathrm{C}}=1 / \tau$ [22], we expect the results reported here for dissipative discontinuous maps to be also applicable to the dissipative version of CSM with $K \gg K_{\mathrm{C}}$.

Acknowledgments. RAS and JAMB acknowledge support form VIEP-BUAP grant MEBJ-EXC13-I and PIFCA grants BUAP-CA-40 and BUAP-CA-169. EDL thanks support from FAPESP, CNPq and FUNDUNESP, Brazilian agency.

\section{References}

[1] A. J. Lichtenberg and M. A. Lieberman, Regular and Chaotic Dynamics (Springer-Verlag, New York, 1992). 


\begin{tabular}{|c|c|c|c|}
\hline & $I_{0} \ll K$ & $\begin{array}{c}I_{0} \gg K \\
\gamma \geq \gamma_{\mathrm{C}}\end{array}$ & $\begin{array}{c}I_{0} \gg K \\
\gamma<\gamma_{\mathrm{C}}\end{array}$ \\
\hline $\begin{array}{l}\left\langle I^{2}\right\rangle \approx I_{0}^{2} \\
\left.I^{2}\right\rangle \propto n K^{2} \\
\left.I^{2}\right\rangle=\frac{1}{2} I_{0}^{2} n^{-1} \gamma^{-1} \\
\left\langle I^{2}\right\rangle \approx I_{\text {sat }}^{2}\end{array}$ & 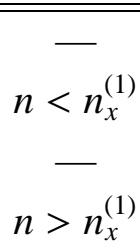 & $\begin{array}{c}n<n_{x}^{(2)} \\
- \\
n_{x}^{(2)}<n<n_{x x}^{(2)} \\
n>n_{x x}^{(2)}\end{array}$ & $\begin{array}{c}n=n_{x}^{(3)} \\
n_{x}^{(3)}<n<n_{x x}^{(3)} \\
- \\
n>n_{x x}^{(3)}\end{array}$ \\
\hline
\end{tabular}

Table 1: Behavior of $\left\langle I^{2}\right\rangle$ for the dissipative discontinuous map of Eq. (5) when $K \gg 1$. We have found that $I_{\text {sat }}^{2} \propto K^{2} \gamma^{-1}, n_{x}^{(1)} \propto \gamma^{-1}, n_{x}^{(2)}=\frac{1}{2} \gamma^{-1}, n_{x}^{(3)} \propto I_{0}^{2} K^{-2}, n_{x x}^{(2)} \propto K^{-2}, n_{x x}^{(3)} \propto \gamma^{-1}$, and $\gamma_{\mathrm{C}} \approx 10^{-4}$.

[2] B. V. Chirikov, Preprint 267, Institute of Nuclear Physics, Novosibirsk (1969) [Engl. Trans., CERN Trans. 71-40 (1971)].

[3] B. V. Chirikov, Phys. Rep. 52, 263 (1979).

[4] J. M. Greene, J. Math. Phys. 20, 1183 (1979).

[5] R. S. MacKay, Physica D 7, 283 (1983).

[6] R. S. MacKay, J. D. Meiss, and I. C. Percival, Physica D 13, 55 (1984).

[7] R. S. MacKay and I. C. Percival, Comm. Math. Phys. 94, 469 (1985).

[8] D. G. Ladeira and J. K. L. Silva, J. Phys. A: Math. Theor. 40, 11467 (2007).

[9] E. D. Leonel, P. V. E. Mcclintock, and J. K. daSilva, Phys. Rev. Lett. 93, 14101 (2004).

[10] D. G. Ladeira and J. K. L. da Silva, Phys. Rev. E 73, 026201 (2006).

[11] O. F. A. Bonfim, Phys. Rev. E 79, 056212 (2009).

[12] O. F. A. Bonfim, Int. J. Bif. Chaos 22, 1250140 (2012).

[13] E. D. Leonel and P. V. E. Mcclintock, J. Phys. A: Math. Gen. 37, 8949 (2004).

[14] E. D. Leonel, Phys. Rev. Lett. 98, 114102 (2007). 
[15] D. G. Ladeira and J. K. L. da Silva, J. Phys. A: Math. Theor. 41, 365101 (2008).

[16] D. F. M. Oliveira and M. Robnik, Phys. Rev. E 83, 026202 (2011).

[17] F. Borgonovi, Phys. Rev. Lett. 80, 4653 (1998).

[18] F. Borgonovi, G. Casati, and B. Li, Phys. Rev. Lett. 77, 4744 (1996).

[19] G. Casati and T. Prosen, Phys. Rev. E 59, R2516 (1999).

[20] G. Casati and T. Prosen, Phys. Rev. Lett. 85, 4261 (2000).

[21] T. Prosen and M. Znidaric, Phys. Rev. Lett. 87, 114101 (2001).

[22] J. A. Mendez-Bermudez, R. Aguilar-Sanchez, Phys. Rev. E 85, 056212 (2012). 